\title{
Presencia de la mujer en los medios de comunicación audiovisuales en Internet
}

\author{
Sonia Núñez Puente \\ María F. Sánchez Hernández \\ Universidad Rey Juan Carlos (España)
}

\section{Resumen}

Se estudia la presencia de la mujer y las repercusiones que tiene en los medios audiovisuales en Internet. Para ello, se ha realizado un recorrido virtual por veinticinco portales dedicados a la radio, al cine y a la televisión. La metodología desarrollada se ha centrado en el análisis de dichas páginas web para conocer los servicios y funciones que se ofrecen desde la Red, así como la difusión a sus destinatarios. Los resultados obtenidos en esta investigación sirven para sistematizar la presencia de la mujer en los medios audiovisuales en Internet y para evaluar la repercusión que dicha presencia ha tenido y tiene en los mismos, así como para conocer las dimensiones de Internet respecto a los medios audiovisuales y a la mujer, sirviendo esta investigación como base para futuros análisis.

Palabras clave: Medios audiovisuales. Presencia y repercusión de la mujer. Internet.

\begin{abstract}
The impact of the presence of women in digital mass media is studied and related to gender studies throughout a virtual trip around 25 web portals focused on radio, movies and television. These web pages has been described and analyzed. It is important to evaluate the presence of women in mass media on the Internet. The results are very relevant for the future of gender studies as a study material as well as a powerful source of information.
\end{abstract}

Keywords: Mass media. Women. Internet.

\section{Introducción}

Los medios de comunicación audiovisuales, desde sus inicios, han formado parte de nuestra vida social, de nuestras costumbres, acompañándonos día a día en los acontecimientos acaecidos e informándonos puntualmente de los sucesos. El cine y la televisión son un arte popular y la forma más conocida de narración del siglo; a veces no solo nos sirven como entretenimiento, sino que nos producen un Scire. 11 : 2 (sep.-dic. 2005) 177-186. ISSN 1135-3761. 
cúmulo de emociones y sensaciones que nos transportan a una realidad paralela o nos muestran a modo de espejo nuestra vida cotidiana reflejando nuestras costumbres.

La sociedad actual, que podría caracterizarse como la sociedad de la comunicación, tiene en los medios de comunicación audiovisuales y en Internet su más fiel imagen. Tanto la televisión como el cine y la radio encuentran en Internet el medio adecuado de expresión y un vehículo preciso de canalización de la comunicación. En este sentido, los sitios web dedicados a la mujer y a su ámbito de actuación son cada vez más numerosos. Son pocos, sin embargo, los trabajos que han intentado echar un poco de luz sobre las interacciones entre las mujeres y su presencia en las nuevas tecnologías, sea en emisoras de radio (1) o en páginas web sobre cine o televisión dedicadas a la mujer.

Gracias a la llegada de los avances tecnológicos podemos desde Internet escuchar emisoras de radio ubicadas en distintos lugares lejos de nuestra conexión, visionar películas sin necesidad de desplazarnos a las salas de cine y disfrutar de servicios on-line televisivos con avanzados sistemas multimedia.

La radio y la televisión como medios de comunicación son vehículos capaces de transportar los mensajes más variados siempre que estén revestidos de un lenguaje audiovisual; pueden, además, convertirse en medios de información, en instrumentos de formación, de evasión o de creación expresiva y, en suma, en difusores de pautas, valores, modelos, y en transmisores de cultura. Son medios de comunicación típicos del siglo XX, particularmente de su segunda mitad, pues requieren mayor complejidad técnica que la prensa y que el cine. Según Fernando Sanabria (1994, pp. 14-16), los medios audiovisuales se hacen imprescindibles en un mundo funcional, plural y universal.

En nuestro trabajo pretendemos aprovechar estos recursos electrónicos para que también nos sirvan como fuentes de información en los estudios de género, recursos en que sin duda la mujer estará como protagonista principal en muchas ocasiones; nos referimos tanto al cine y la televisión como a emisoras y programas de radio especializados o destinados a mujeres.

Hoy día, la tecnología digital ha cambiado la forma de trabajo, de comunicación y de entretenimiento entre personas, sobrepasando las barreras geográficas y brindándonos oportunidades informativas y de ocio desde lugares que geográficamente se encuentran distanciados.

Dentro de un campo de estudio tan impreciso como la presencia y repercusión de las mujeres en Internet, cabe destacar que la Red ha creado un nuevo mundo virtual y paralelo que es una suerte de imagen de la sociedad igual que los demás medios de comunicación. Por ello, también en el llamado ciberespacio se consolidan y afianzan los estereotipos de género. Estos estereotipos se presentan en los me- 
dios de comunicación audiovisuales en Internet de tres formas bien diferenciadas: como imagen de la mujer creada para los hombres, como imagen de la mujer creada para las mujeres y como imagen de la mujer creada por las propias mujeres.

\section{Metodología}

Teniendo como meta principal la sistematización de la presencia de la mujer y de sus estereotipos en los medios audiovisuales en Internet, hemos llevado a cabo las siguientes tareas: (I) búsqueda y localización de las fuentes de información adecuadas a nuestro estudio; (II) conocer su contenido y difusión; (III) establecer un análisis comparativo de la presencia y repercusión de la mujer en los distintos medios audiovisuales en Internet; (IV) ofrecer una guía tanto de la presencia de la mujer como de la repercusión de la misma en los medios audiovisuales en Internet.

\section{Análisis de los portales estudiados}

\subsection{Ministerio de Cultura. Filmoteca}

La Filmoteca Española es una Subdirección General del Instituto de la Cinematografía y de las Artes Audiovisuales. Tiene como misión recuperar, investigar y conservar el patrimonio cinematográfico y promover su conocimiento. Forma parte de la Federación Internacional de Archivos Fílmicos (FIAF), que desde 1956 otorga a estas instituciones el carácter de archivos históricos. También investiga, recupera y restaura el patrimonio cinematográfico español. Desde sus distintos enlaces podemos recuperar información relacionada con la mujer en el cine a través de sus bases de datos visionando dichos archivos o pudiéndolos localizar en formato texto.

\subsection{Radio Paca}

Ofrece un espacio de representación y difusión sobre las ideas y acciones de las mujeres contribuyendo al diálogo y presencia femeninos en el mundo a través de una programación específica para mujeres con secciones dedicadas a música, magazines, tertulias, informativos, etcétera.

\subsection{Nuestro cine.com}

Web donde se puede encontrar información sobre cine, filmografías, biografías de directores, carteles, etcétera. En el apartado dedicado a la mujer, encontramos la incorporación de las mujeres como directoras, la cual se considera reciente ya que hasta hace relativamente pocos años era una "misión imposible" para ellas. Destacamos que la primera mujer directora en términos absolutos fue Alice Guy, que de secretaria de la Gaumont pasó a directora, de la manera en que en aquella época (a partir de 1896) se dirigía, es decir, se cogía la cámara y se filmaba. También aparece un listado de películas en cuyo título se incluye el término mujer/mujeres.

Scire. $11: 2$ (sep.-dic. 2005) 177-186. ISSN 1135-3761. 


\subsection{Radio Feminista}

A través de esta web, podemos escuchar la emisora Radio Feminista, que a pesar de tener carácter internacional refleja la identidad latinoamericana y caribeña. Sus objetivos son, entre otros, promover la presencia de las mujeres en los medios de comunicación; impulsar la transmisión de imágenes no estereotipadas de las mujeres en los medios de comunicación, además de reconocer y respetar la diversidad de las mujeres; fomentar las comunicaciones no sexistas y los derechos humanos de las mujeres, y extender la democratización de las comunicaciones, el acceso y la capacitación de las mujeres en las nuevas tecnologías.

\subsection{La ciudad de las mujeres en la red}

Esta página se compone de varias secciones, entre ellas una de cine/multimedia, donde existen, además de artículos, foros de discusión, siempre abordando el tema de la mujer.

\subsection{Radio Televisión Española}

A través de link del Instituto Oficial de Radio Televisión Española, accedemos al informe titulado «Representación de la violencia de género en los informativos de TVE: conclusiones», donde se señala que el 12,5\% de las mujeres entrevistadas pertenecen a un grupo de cuya profesión no se informa, a quienes les siguen las políticas con un 3,2\% y en tercer lugar las estudiantes con un 2,6\%. Los hombres en TVE 1 eran representados con posiciones de mayor reconocimiento social y en papeles más variados que las mujeres; en el telediario de TVE 2 (el de máxima audiencia de los tres analizados), del total de noticias relacionadas con la mujer un $2 \%$ corresponde a violencia de género y señalamos como dato interesante de este informe que en el Foro Económico Mundial, donde se analiza la situación de la mujer en 58 países, se ha utilizado como planos-recurso a mujeres en una amplia variedad de papeles: trabajadoras de una fábrica de confección, enfermeras, pediatras, limpiadoras, etcétera, generalmente relacionados con los roles tradicionales asignados a la mujer, y solamente una arquitecta.

\subsection{El mundo.com}

Este portal depende del diario El Mundo. En él se recogen ideas de distintos cineastas sobre el panorama español, cuyo objetivo es la creación de un proyecto titulado Hay motivo, integrado por varios cortometrajes que sirven de crítica a la sociedad actual. Como se puede apreciar, la presencia femenina es bastante inferior a la masculina.

\subsection{El Periódico Feminista de Mujeres en Red}

Se trata de una publicación periódica en la Red. Si realizamos una búsqueda sobre mujer y cine encontraremos información relativa a eventos cinematográfi- 
cos, ya sean películas, seminarios, certámenes, etcétera, con amplia información sobre cada uno de ellos.

\subsection{Les Pénélopes}

A través de la red francesa Pénélopes-Ciberfemmes se puede visualizar la televisión interactiva dedicada a temas de género, que ofrece la posibilidad de ver los programas en diferido. También un equipo internacional de activistas e investigadoras de la producción independiente recopila Datafighters, a modo de narración en vídeo con una introducción contextual y una duración de unos 150 minutos como máximo. Traducidos a inglés, árabe, español, francés, portugués e italiano, son videodocumentales que presentan la "brecha digital" norte-sur y la desigualdad de género, entre otras temáticas.

\subsection{Buscacine.com}

Contiene un motor de búsqueda que nos remite a numerosos enlaces con información acerca de la mujer y el mundo audiovisual, como por ejemplo la Muestra Internacional de Films de Mujeres en Barcelona o la descripción del papel representado por mujeres como en las películas El diario de Bridget Jones, Erin Brockovich, Van Wilder Animal Party (web oficial en España de esta película protagonizada por Tara Reid), Crueles intenciones, American Pie, El gran Lebowsky, Dr. T y las mujeres, Woman On Top (Las mujeres arriba, protagonizada por Penélope Cruz), además de enlaces con telenovelas donde la mujer también aparece como protagonista principal.

\subsection{AMECO. Nodo50}

En esta web podemos localizar a modo de directorio los siguientes apartados: asociaciones y grupos de mujeres periodistas; redes y agencias de distribución de información sobre las mujeres en Internet; revistas, boletines, comunidades virtuales en Internet y por países, medios de comunicación de mujeres (revistas impresas y programas de radio).

\subsection{Nuestro cine}

Podemos encontrar en esta página un enlace dedicado a anuncios audiovisuales que permite seleccionar a través de su buscador spots publicitarios de mujeres o sobre mujeres; son más de 2300 anuncios que representan a un total de 1300 marcas. Se ofrece la posibilidad de visionarlos, y son gratuitos e internacionales. Este portal puede servir para llevar a cabo un estudio desde el punto de vista de género y publicidad.

\subsection{Ministerio de Trabajo y Asuntos Sociales. Instituto de la Mujer}

El Centro de Documentación del Instituto de la Mujer, dependiente del Ministerio de Trabajo y Asuntos Sociales a través de la Secretaría General de Políticas Scire. 11 : 2 (sep.-dic. 2005) 177-186. ISSN 1135-3761. 
de Igualdad tiene como finalidad promover y fomentar las condiciones que posibiliten la igualdad social de ambos sexos. Esta unidad de información alberga un rico material audiovisual relacionando el papel de la mujer con distintas temáticas (medicina, educación, política, trabajo, violencia de género, etcétera).

\subsection{Mascine.com}

En esta URL podemos localizar un enlace dedicado a "mujer y cine", donde encontraremos biografías de actrices que han destacado en la historia del séptimo arte, como Marilyn Monroe, Andrey Hepburn, Greta Garbo, Vivien Leigh o Lauren Bacall. También hay un espacio destinado al cine francés, español, estadounidense, británico y mexicano. Hay que señalar que en el apartado de directores de cine solamente se recogen las trayectorias profesionales de hombres.

\subsection{Webpilots.net}

En su enlace dedicado a cultura y en concreto el apartado de cine nos ofrece información sobre actores, directores y películas que han hecho historia, como Marlene Dietrich; hay un apartado de actores y directores que no han obtenido ningún premio Oscar pero que sí han ocupado un lugar importante en el cine. En el apartado dedicado a España destaca la actriz Penélope Cruz.

\subsection{CulturaliaNet}

Actúa como un lugar informativo donde se pueden realizar tanto búsquedas como navegación; siempre remite a páginas con información, cada una de las cuales cuenta con diferentes enlaces en su parte inferior, entre los que destacamos "Buscar la película". También se puede comprar la película en DVD o VHS, solicitar su banda sonora en $\mathrm{CD}$ o leer un artículo especializado, ya que se oferta más de medio centenar.

\subsection{Antena 3}

Desde la página principal de Antena 3 Televisión podemos acceder a distintos links dedicados a la mujer, donde encontraremos portales de sitios oficiales (institutos de igualdad dependientes de la Administración Pública), foros, eventos y revistas especializadas en el género, donde la presencia femenina es notable.

\subsection{Worldonline.es}

Se trata de un artículo donde se expone un análisis sobre los cómics y los dibujos animados, reflejando que tanto los hombres como las mujeres son auténticas víctimas del estereotipo sexual, aunque la representación de las mujeres es mucho más baja que la de los hombres; por ejemplo, la exclusión femenina aparece en dibujos tales como los de Bugs Bunny. 


\subsection{Producciones Nicobis}

Podemos localizar un enlace con cuatro vídeos sobre mujeres bolivianas donde trata de la historia del movimiento de mujeres en Bolivia durante el siglo XX, junto con biografías de mujeres del mismo siglo que han destacado internacionalmente.

\subsection{Facua}

Desde este portal se denuncia a las empresas anunciadoras que difunden publicidad ofensiva para la imagen de la mujer en distintos medios audiovisuales, ya que la Ley General de Publicidad de 1988 en España prohíbe todo anuncio "que atente contra la dignidad de la persona o vulnere los valores o derechos reconocidos en la Constitución, especialmente en lo que se refiere a la infancia, la juventud y la mujer".

\subsection{Revista Qué Pasa}

Es una revista difundida también a través de la Red; la hemos seleccionado porque en uno de sus artículos, publicado el 30 de abril de 2004, ofrece un balance sobre la situación actual del cine hasta la fecha, con referencias al género.

\subsection{Revista Consumer}

En un enlace de la revista, al cual accedemos desde su página principal, se publica una entrevista realizada a la directora de cine Chus Gutiérrez, que analiza su trayectoria profesional, así como la situación de las mujeres en el mundo del cine.

\subsection{Fotograma}

Utilizando su buscador encontramos en este enlace, más de medio centenar de links donde localizamos la presencia de la mujer en películas, no solo como actrices (generalmente en papeles secundarios), sino también como directoras. Así mismo, se refleja que en muchas ocasiones los premios más prestigiosos también se han olvidado de ellas.

\subsection{Femina Interactive}

Dispone de un enlace dedicado a "las mujeres y el cine", donde, gracias a su amplio menú, podemos localizar información sobre cineastas femeninas, un calendario de eventos cinematográficos, foros, magazines, anuncios, etcétera.

\subsection{Telecinco}

Desde la página principal de Telecinco podemos realizar una búsqueda sobre "mujer y programas". El resultado son más de cien enlaces donde destaca la presencia de los temas relacionados con el ámbito femenino, tales como el papel de las mujeres en televisión, publicidad, revistas, recursos electrónicos y audiovisuales, noticias sobre violencia de género, acoso sexual, foros, trabajo, etcétera. 


\section{Conclusiones}

Este estudio nos ha servido principalmente para conocer las dimensiones de la presencia de la mujer en los distintos portales destinados a ofrecer información sobre la relación explícita entre esta y los medios audiovisuales, así como para evaluar la repercusión que dicha presencia ha tenido y tiene en los mismos.

Los portales analizados los ofrecemos como base para futuros análisis y para sistematizar cómo se presenta la mujer, y la imagen posterior que de ella se ofrece en los medios audiovisuales en Internet, centrándonos, sobre todo, en aquellos portales dedicados al cine, la radio, la publicidad, la televisión y los periódicos feministas.

Hemos apreciado que en algunas páginas web la presencia femenina es destacable. No obstante, en nuestra opinión, al igual que en otros ámbitos de la vida pública, la mujer aparece discriminada o infravalorada. Sirvan de ejemplo, en este sentido, los informativos de televisión donde en muchas ocasiones los planosrecurso que se utilizan representan a una mujer vinculada a los roles tradicionales de madre o esposa y, por el contrario, los hombres siempre aparecen ocupando puestos de trabajo más relevantes.

También observamos que la mujer ha tenido mayores dificultades de acceso al medio audiovisual, especialmente por lo que respecta a la representación de papeles de protagonista en películas o a la ocupación de altos cargos dentro de las empresas audiovisuales. Y todo ello a pesar de que el 62\% del alumnado universitario es femenino. Constatamos, de esta manera, en este apartado, una creciente desigualdad en las oportunidades de desempeño de roles preeminentes en la sociedad, y esto se ve reflejado en la industria cinematográfica. En este sentido, algunos de los portales analizados mantienen como línea fundamental de sus contenidos la necesidad de sensibilizar, reivindicar e informar acerca de la violencia simbólica. Esto es, se recurre a la denuncia de la ausencia de roles primordiales en el cine. Desde las páginas web analizadas se pretende, de esa forma, ofrecer una visión crítica sobre la presencia de las mujeres en el cine.

En cuanto a la publicidad, cabe señalar que se sigue utilizando el "cuerpo femenino" como reclamo de numerosas marcas conocidas como "reclamo de consumo", hiriendo la dignidad de la mujer. A este respecto existen, y se muestran en las páginas web estudiadas, recursos de regulación sobre la imagen de la mujer en la publicidad, como los que mantiene activos el Instituto de la Mujer en su página.

Por lo que hace a las emisoras de radio que dedican su programación a las mujeres, han sido muy escasas. No obstante, existen emisoras que promueven una conciencia clara y equilibrada de los derechos humanos y temas globales de desarrollo. La programación de este tipo de emisoras gira fundamentalmente en torno a programas de debate, informativos y de asesoramiento y consejos.

Scire. 11 : 2 (sep.-dic. 2005) 177-186. ISSN 1135-3761. 
Por último, se hace necesario destacar que los institutos dependientes de la Administración Pública promueven el papel de la mujer con medios audiovisuales educativos. Estas medidas se dirigen a intentar alcanzar una igualdad para todos, para lo que las tecnologías de la información y la comunicación, y en concreto Internet, ofrecen grandes posibilidades.

\section{Notas}

(1) Uno de los grandes avances de las TIC es la posibilidad de escuchar la emisora de radio desde Internet; el avance en este aspecto ha sido enorme, según Cebrián (1995, p. 33) y Arboledas (1995, p. 42). La primera radiodifusión en España tuvo lugar en 1912 gracias a Matías Balsera; se situó una emisora en el Palacio de Comunicaciones y se efectuaron las primeras transmisiones de la ópera desde el Teatro Real de Madrid, además de conciertos de la Banda Municipal desde el parque del Retiro.

\section{Referencias}

Arboledas, Luis (1995). Radiofonistas, predicadores y pinchadiscos. Granada: Comares, 1995.

Cebrián Herreros, Mariano (1995). Información radiofónica: mediación técnica, tratamiento y programación. Madrid: Síntesis, 1995.

Sanabria, Fernando (1994). Información audiovisual, teoría y técnica de la información radiofónica y televisiva. Barcelona: Bosch Comunicación, 1994.

\section{Anexo. Guía de recursos en línea sobre la presencia de la mujer en los medios audiovisuales}

1. Ameco. Nodo 50 (2005). URL: <http://www.nodo50.org/ameco/enlaces.html>. Consultado: 2005-03-11.

2. Antena 3 (2005). URL: <http://www.antena3.com/a3tv_2004/espotting/buscaespotting. html?keyword=programas\%20y\%20mujer $>$. Consultado: 2005-03-11.

3. Audiored, S. P. C. (2006). URL: <http://web-mujer.anuncios-radio.com>. Consultado: 2005-03-14.

4. Busca cine (1999-2006). URL: <http://www.buscacine.com>. Consultado: 2005-03-01.

5. CanalWeb.net (2005). URL: <http://www.canalweb.net/cwsite/cw/default.asp?une= cyberfemmes>. Consultado: 2005-03-10

6. Cine por la red (2005). URL: <http://www.porlared.com/cgi-bin/perlfect/search/ search.pl?q=mujer $>$. Consultado: 2005-03-15.

7. Facua anuncios (2005). URL: <http://www.facua.org/facuaanuncios/mujerypublicidad/ mujerypublicidad.htm>. Consultado: 2005-03-01.

8. Fotograma.com (2005). URL: <http://www.fotograma.com/buscar/index.pl?query=mujer>. Consultado: 2005-03-23.

9. La ciudad de las mujeres en red. Artefinal Studio (2001). URL: <http://www.e-leusis.net>. Consultado: 2005-03-21.

Scire. $11: 2$ (sep.-dic. 2005) 177-186. ISSN 1135-3761. 
10. Culturalianet (2005). URL: <http://www.culturalianet.com>. Consultado: 2005-03-08.

11. Fundación Eroski. Revista Consumer (2005). URL: <www.revista.consumer.es>. Consultado: 2005-03-14.

12. Mascine (2005). URL: <http://mascine.webcindario.com>. Consultado: 2005-03-01.

13. Mascine (2005). URL: <http://www.es.webpilots.net/arte_cultura/cine/cine.phtml>. Consultado: 2005-03-01.

14. Misterio de Cultura. Filmoteca (2005). URL: <http://www.mcu.es/cine/film/ filmoteca.jsp>. Consultado: 2005-03-20.

15. Ministerio de Trabajo y Asuntos Sociales. Secretaría General de Políticas de Igualdad. Instituto de la Mujer. Centro de Documentación (2005).

URL: <http://www.mtas.es/mujer/servicios/centro_documentacion/cendoc.htm>. Consultado: 2005-03-20.

16. Nuestro cine (2005). URL: <http://www.nuestrocine.com>. Consultado: 2005-03-04.

17. El Periódico Feminista de Mujeres en Red (2005). URL: <http://www.mujeresenred. net/news>. Consultado: 2005-03-24.

18. Radio Paca. WordPress and Blue Memories by iqwolf.Entries (RSS) and Comments (RSS) (2005). URL: <http://www.radiopaca.org>. Consultado: 2005-03-26.

19. Radio Internacional Feminista. FIRE (2005). URL: <http://www.fire.or.cr>. Consultado: 2005-03-25.

20. Radio Televisión Española. Instituto Oficial de Radio Televisión Española (2005). URL: $<$ http://www.rtve.es/oficial/iortv/SegundoInforme.pdf > Consultado: 2005-03-10.

21. Revista Qué Pasa. Consorcio Periodístico de Chile, S. A. (2005).

URL: <www.quepasa.cl>. Consultado: 2005-03-10.

22. Telecinco. Europortal Jumpy España, S. A. (2005). URL: <http://www.telecinco.es>. Consultado: 2005-03-23.

23. Webgrrls International, Inc. Sites for, by and about women (2003). URL: <http://www.femina.com>. Consultado: 2005-03-26.

24. La web del cine español (2005). URL: <www.nuestrocine.com>. Consultado: 200503-01.

25. Les Pénélopes (2005). URL: <http://www.penelopes.org/Espagnol/xbreve.php3 ?id_rubrique=13>. Consultado: 2005-03-14.

26. Utopos.org (2005). URL: <http://www.utopos.org/Cine/Produc/Nicobis.htm>. Consultado: 2005-03-05.

27. Worldonline.es (2005). URL: <http://home.worldonline.es/hekokole/genero.htm>. Consultado: 2005-03-15.

28. Webpilots España (2005). URL: <http://www.es.webpilots.net/arte_cultura/cine/ cine.phtml>. Consultado: 2005-03-10. 